\title{
LP-INDEX: Explore the Best Practice of LPWAN Technologies in Smart City
}

\author{
Hao Wang \\ Department of Electrical Engineering \\ City University of Hong Kong \\ Hong Kong, China \\ hwang272-c@my.cityu.edu.hk \\ Yaqing $\mathrm{He}$ \\ Department of Electrical Engineering \\ City University of Hong Kong \\ Hong Kong, China \\ yaqinghe2-c@my.cityu.edu.hk \\ Chun Sing Lai \\ Department of Electronic and Computer \\ Engineering \\ Brunel University London \\ London, UK \\ chunsing.lai@brunel.ac.uk
}

\author{
Yucheng Liu \\ Department of Electrical Engineering \\ City University of Hong Kong \\ Hong Kong, China \\ yucliu4-c@my.cityu.edu.hk \\ Kim Fung Tsang \\ Department of Electrical Engineering \\ City University of Hong Kong \\ Hong Kong, China \\ ee330015@cityu.edu.hk
}

\author{
Yang Wei \\ Department of Electrical Engineering \\ City University of Hong Kong \\ Hong Kong, China \\ ywei22-c@my.cityu.edu.hk \\ Loi Lei Lai \\ Department of Electrical Engineering \\ Guangdong University of Technology \\ Guangzhou, China \\ 1.1.lai@ieee.org
}

\begin{abstract}
Internet of Things (IoT) based infrastructures and applications are essential parts in smart city establishment. The low power wide area network (LPWAN) plays a key role in IoT techniques due to the characteristics of wide coverage and low power consumption. However, it is hard to decide which one of the LPWAN techniques to be implemented in a specific application for best practice. Considering this, we discussed the main characteristics of the three popular LPWAN technologies, LoRaWAN, NB-IoT, and Sigfox, and proposed an LP-INDEX to weighting them according to application requirements. To further distinguish their difference, a comparison test based on parking detection sensors using the three different techniques was implemented as a use case.
\end{abstract}

Keywords- Smart City, LPWAN, LoRaWAN, NB-IoT, Sigfox, LP-INDEX

\section{I.INTRODUCTION}

The Internet of Things (IoT) technology has been a fundamental one that facilitates the construction of smart applications, aiming at improving the production efficiency and human living quality, such as smart city, smart industry, smart agriculture, etc. Larger coverage of wireless technology is required to achieve the best performance of IoT applications which are in the trend of increase these days. The Low Power Wide Area Network (LPWAN) technologies are suitable alternatives to fulfill the requirements. The LPWAN is proposed to replenish the vacancy of current wireless technologies including Wi-Fi and Bluetooth, which provide service in a short distance with high bandwidth. The LPWAN earns its popularity around the world due to its low power, long-distance, and low cost in communications. It could provide up to $10-40 \mathrm{~km}$ in rural zones and $1-5 \mathrm{~km}$ in urban zones with high energy efficiency [1]. The cost of a radio chipset is about 2 USD and operating cost per device is about 1 USD for one year [2]. These characteristics of LPWAN had attracted researchers to perform numerous studies on applications, and indoor and outdoor evaluation of LPWAN. Lots of LPWAN applications are developed accordingly and they have proved the reliability and appropriateness of LPWAN technologies. LPWAN technology is one of the most suitable choices for these applications which needs to transmit data over long distances. Currently, popular LPWAN technologies include Sigfox [3], LoRaWAN [4], NB-IoT [5].

The governments all over the world are pacing rapidly on smart city initiative and LPWAN deployment is, nonetheless, one of the focuses of the Government. Since there are various technologies for LPWAN and a global IEEE standard is not in place, it is desperate to develop a new standard to aid sensor interoperability and combat IoT cyber threat. This investigation intends to compile best practices for the technologies to achieve the best performance. To investigate the appropriate technology to be applied to different IoT applications respectively, a guideline on the usage of these technologies should be introduced. Thus, a guideline would be proposed for the best practices of LPWAN to promote IoT development. In this paper, LPWAN technologies including Sigfox, LoRaWAN, and NB-IoT were evaluated. Technical features among these technologies would be compared. Then the success factors such as coverage, latency, etc would be compared to further define the characteristics of each technology. At last, an IoT index would be developed, named LP-INDEX for LPWAN technologies to evaluate their performance in different scenarios. The LPWA-index evaluates the LPWAN in different aspects including Latency, Data Capacity, Power and Cost, Coverage, Scalability, and Security, and it provides a final score for each LPWAN technique based on application requirements. Besides, the LPWAN network was applied to better explore the characteristics through experiments.

The contributions are summarized as follows:

1. An LPWAN index for the evaluation of LPWAN, LPINDEX, is proposed to provide a guideline for finding the best practices of LPWAN. The developed LP-INDEX guides LPWAN based applications and leads the LPWAN deployment in smart city construction. 
2. Testing for three LPWAN comparisons based on a parking detection sensor was implemented as a use case to illustrate how LP-INDEX works. To the best of our knowledge, there are no similar testings in related work.

The remaining of this paper is organized as follows: Section II summarizes the state-of-art works in LPWAN. Section III introduces the main characteristics of the three LPWAN techniques. Section IV explains how LP-INDEX is established. Section V describes the experimental results and gives a discussion. Finally, Section VI provides a conclusion and future work.

\section{RELATED WORK}

The unique advantages of LPWAN have attracted a lot of researchers to perform further studies on them. This section discusses the previous studies related to the conducted LPWAN technologies.

Nowadays numerous researchers concentrate on LPWAN-based applications, such as monitoring, localization, sensing, etc. These works contribute to facilitating the implementation of LPWAN technologies in reality and attract more attention from investigators. Meanwhile, since the development of LPWAN is still in its infancy, more researches focus on the evaluation of LPWANs.

Except for the works listed above which evaluate LPWANs technologies individually, some researchers studied them together and compared various LPWANs in different terms. In a related work [6], the authors discussed different LPWAN technologies in mac-layer. They compared Sigfox, LoRa, NB-IoT with their features of the radio frequency and evaluated them in terms of packet error rate. In another study [7], the authors explored the main features of LoRa and Sigfox and conducted a coverage estimation test case. They also performed experiments to estimate the largest coverage of LPWAN. Besides, in [8] the authors reviewed the main LPWANs and list their technical features such as modulation, frequency, bandwidth, etc. The authors further compared them in terms of performance factors such as coverage, latency, etc. It was available to know the strengths and drawbacks of different LPWANs from this paper, but it lacked for experiments and original algorithm.

The mentioned works all contribute to the research of LPWAN technology. However, among all current researches, studies related to the best practices of LPWAN are lacking behind. The lack of related guideline on LPWAN choice based on specific scenarios causes a confusing for users. For decision-makers, it is hard for them to select the most applicable one from so many LPWAN technologies. Considering this, we proposed an IEEE P2668 based LPWAN index to evaluate different LPWANs in various applications.

\section{PRELIMINARIES}

\section{A. LoRaWAN}

LoRa is a physical layer technology that modulates the signals in the sub-GHz Industrial, Scientific and Medical (ISM) band using a proprietary spread spectrum technique [4]. Similar to Sigfox, LoRa uses unlicensed ISM bands,
923.2 MHz in Hong Kong. The chirp spread spectrum (CSS) modulation, which spreads a narrow-band signal over a wider channel bandwidth is applied by LoRa. This results in a signal with low noise levels, high interference resilience, and it is difficult to be detected or jammed. One unique feature of LoRaWAN is the flexible establishment without Internet Service Providers (ISP), which makes it cost less and has an extra chance to ensure security. It replaces the cost of subscription and management fees by gateway purchase and installation fees. Though the cost of the LoRa gateway is not a small number, the whole cost of LoRaWAN network establishment is decreased if the cheap gateways are selected. Besides, the flexibility brought by the private establishment of LoRaWAN network becomes another advantage. Comparing to other LPWANs whose base stations are fixed, it is more convenient to provide a good cover for applications. Users can deploy the gateway to the most appropriate site, which enables most devices to send and receive high-quality signals. In addition, security can be further ensured if users do not connect to the public network. The ability of flexible network establishment is a unique advantage of LoRa. Another unique feature of LoRa is that it adopts an adaptable data rate (ADR) algorithm through six spreading factors (SF7 to SF12) to make tradeoff with coverage. A higher spreading factor indicates larger coverage at the expense of a lower data rate. The LoRa data rate varies between $300 \mathrm{bps}$ and $50 \mathrm{kbps}$ based on spreading factor and channel bandwidth. Also, messages transmitted through various spreading factors can be received simultaneously by LoRa base stations. In 2015, LoRaWAN, a LoRa-based communication protocol is first proposed by LoRa-Alliance. Based on LoRaWAN, signals transmitted by end devices can be received by all the base stations which cover. The success transmission ratio is thus increased due to this redundant reception. Since multiple base stations are needed nearby, this capability incurs additional network deployment costs. However, there are more advantages to this disadvantage. For example, a time to arrival (TDOA) based localization technique was developed, supported by very precise time synchronization between different base stations. Therefore, LoRaWAN is applied to localization by analyzing the strength of the signal received from the same terminal device. This feature also prevents handoffs because the terminal always sends signals to all available base stations, so no handoffs are required.

\section{B. NB-IoT}

NB-IoT is specified in Release 13 of the Third Generation Partnership Project (3GPP) as a Narrow Band IoT technology in 2016 [5]. NB-IoT occupies a frequency bandwidth of $200 \mathrm{kHz}$, which corresponds to one resource block in Global System for Mobile Communications (GSM) and Long Term Evolution (LTE) transmission. NB-IoT is developed based on the LTE protocol. It can be seen as a simple version of LTE without complex protocol functionalities. It employs the quadrature phase-shift keying modulation (QPSK). The NB-IoT is further improved to be more suitable for IoT applications. When the LTE backend transmits signals to available end devices in a cell, it will decrease these necessary steps adopted in LTE protocols such as channel quality monitoring, carrier aggregation, and dual connectivity, which are unnecessary for IoT applications. It decreases the power 
and resource consumption to a minimum. For each cell, at most 100,000 devices are allowed to connect to the NB-IoT carries. It could break through the number limit by adding more carries. There are 12 subcarriers inside the channel and each subcarrier is separated by $15 \mathrm{kHz}$. NB-IoT uses single carrier frequency division multiple access (SC-FDMA) modulation and orthogonal frequency division multiple access (OFDM) modulation for uplink and downlink transmissions. This makes large connections and reliable two-way communication possible. Since it is deployed in the licensed band, NB-IoT has a relatively large throughput, which enables device firmware to be updated over the air. The NB-IoT uplink effective data rate is $0.5-140 \mathrm{kbps}$, and the downlink effective data rate is $0.3-125 \mathrm{kbps}$. In addition, NB-IoT benefits from a licensed band with no duty cycle restrictions. But the disadvantage is the high deployment cost of narrowband IoT. NB-IoT has three network deployment methods: in-band, guard-band, and stand-alone [3]. NB-IoT spectrum is deployed inside the LTE spectrum band with $180 \mathrm{kHz}$ bandwidth which is one resource block of an LTE channel in in-band mode. For guard-band deployment, $180 \mathrm{kHz}$ NB-IoT spectrum is placed by ISPs in the existing LTE signal's guard bands. NB-IoT spectrum can also be entirely separated from the existing LTE spectrum in standalone solution.

\section{Sigfox}

Sigfox is an LPWAN network operator that connects each customer with end-to-end service. The Sigfox base stations are deployed with software-defined radios. They are connected to the backend server through an IP-based network. The protocol that is applied by end devices to connect to these base stations is binary phase-shift keying (BPSK) modulation [3]. The application of the ultra-narrow band makes bandwidth more efficient and noise levels lower. This further leads to lower power consumption and cost. In Sigfox, data from the base stations to the end devices as downlink communication will be sent following an uplink communication. Due to the number of messages in the downlink, it is impossible to acknowledge each uplink message. Then, the uplink communication reliability is ensured by time and frequency diversity, and transmission duplication. Each end-device message will be transmitted three times (by default) over different frequency channels. Sigfox divides the global region into 7 regions from $\mathrm{RC} 1$ to RC7. Each region specifies different operating rules for the Sigfox device, including frequency range, data rate, multiple access mechanisms, and hardware specifications. Sigfox uses a lightweight protocol for short message transmission to ensure low power consumption. This lightweight protocol typically limits up to 140 upstream transfers per day, with a maximum payload of 12 bytes and a maximum downstream transfer of 28 bytes, and is used only for upstream identification. However, this prevents the Sigfox network from responding to most upstream messages. The frequencyhopping used in RC4 allows each message frame to be broadcast three times on a different frequency. Besides, the second send can be executed after 20 seconds. Therefore, the delivery of packaging can be ensured. Sigfox uses $100 \mathrm{~Hz}$ bandwidth ultra-narrow band (UNB) modulation to achieve ultra-low noise level. Thus, the long-distance transmission and high sensitivity at the receiver end can be realized.

\section{LP-INDEX FOR BEST PRACTICES OF LPWAN TECHNOLOGIES}

The IEEE Standard Working Group P2668 [9] is developing an indicator, namely the IoT Index (IDex), to measure the maturity of IoT objects and produce quantifiable evaluation results. The IoT objects refer to a variety of physical and cyber objects in the IoT ecosystem, such as devices, machines, networks, systems, services, and infrastructure. The IDex indicates the integral performance of IoT objects pertinent to their applications. An IoT object can have different IDex levels when it is implemented to various applications and scenarios. For instance, a wireless protocol can achieve a high IDex level in non-critical use cases, but it may attain a low IDex level in critical use cases. With the salient features offered by the IDex, the IoT stakeholders can compare, adapt, and develop IoT solutions that fit the applications' requirements and comply with the global standards. An example of the IDex use case on evaluating is provided as follows:

Low-power Wide-area Network (LPWAN) is an emerging wireless technology that aims to address the IoT's needs for high-scalability, low-power, and large-coverage connections. As a trade-off, the LPWANs are usually latency-tolerance and lack of advanced security measures. For IDex applications in LPWAN, we proposed a sub-trail named LP-INDEX.

The LP-INDEX can manifest high-level guidance on the wise selection (and integration) of IoT solutions. It evaluates the LPWAN based applications based on the following aspects: (1) Latency, (2) Data Capacity, (3) Power and Cost, (4) Coverage, (5) Scalability, and (6) Security. For each application, a set of preferences can be defined according to the application requirement in terms of these six aspects. For instance, an intelligent transport system requires 10 to 50 Mbps of data rate, $10 \mathrm{~ms}$ of latency, and a connection density of $4000 / \mathrm{km} 2$. Then, weightings are distributed for all the aspects. The weightings are considered mainly based on the application requirement. Special requirements of users will be focused in particular. At the same, evaluations are made based on these preferences, and representative grades for the six aspects are made. Finally, a total score is given based on Equation (1):

$$
S_{\text {total }}=\sum_{1}^{i} w_{i} S_{i}
$$

In Equation (1), $\boldsymbol{w}$ and $\boldsymbol{S}$ represent the weighting and evaluation score for each preference.

The following sections introduce the specific content for the six considered aspects of LPWAN.

\section{A. Latency}

The latency indicates by the time it takes for a request to travel from the sender to the receiver and for the receiver to process that request. In other words, the round trip time from the sender to the server. It is desirable to have this time to remain as close to zero as possible, however, in practice, there are constraints preventing the latency times to be zero but we try our best to keep it low. Therefore, it is an essential parameter for these applications with restricted limitations in real-time. However, LPWAN generally does not perform well on latency due to the network characteristic. Thus, other 
wireless networks are recommended to replace LPWAN for those applications with ultra-low latency requirements. Though the low latency is not the advantage of LPWAN, it still deserves to be compared among different LPWANs. Some applications for LPWAN still require as low latency as possible due to the application requirement.

\section{B. Data Capacity}

Data capacity reflects the available transmitted message in one turn, which is essential in such applications with a large packet size requirement. Data rate and payload length are thought to be the main parameters to reflect the performance of data capacity in this part. Higher data rates enable more devices to transmit more information, thus improving data transmission capacity. The larger the payload is, the more information is sent around. Due to the various LPWAN protocols, the three LPWANs have various data capacity characteristics. It is worth mentioning that Sigfox has the largest data transfer limitation daily. This may bring a negative effect for these applications in asking for frequent communication.

\section{Power and Cost}

Low power is one of the main characteristics of the three LPWAN technologies. The performance of power consumption is critical for battery-powered IoT end devices because unrealistic costs would be incurred by frequently replacing large network batteries. Sensors in LPWAN applications, such as temperature and humidity sensors, are dedicated to maintaining minimum power consumption to extend the battery life of sensor devices. Thus, the power consumption for different operation modes should be considered. In addition, the working period of the different patterns determines the duty cycle of each technology. For example, longer sleep patterns can reduce power consumption. Among these three, NB-IoT consumes a larger power due to its communication protocol. Unlike, Sigfox and NB-IoT, LoRaWAN provides an extra choice-Class $\mathrm{C}$ with more power consumption but supporting bidirectional communication.

The cost is the basic issue that is considered in all applications. Usually, the user should choose the most suitable one within the budget. In terms of cost, several parameters including sensor cost, gateway cost, installation cost, subscription cost, management cost, and sensor recurring cost should be considered. As discussed previously, usage fees are needed for Sigfox and NB-IoT service, while for LoRaWAN it is free. For LoRaWAN, since the network could be established freely, the additional cost is generated for LoRaWAN gateways. Besides, the deployment fee, the sensor fee, the maintenance fee of the three LPWAN technologies should be considered in practice.

\section{Coverage}

The coverage indicates the operation range of the LPWAN technology. In general, the large coverage is a common characteristic of LPWANs due to their protocol. This characteristic fulfills the gap of the traditional wireless network. The traditional wireless network needs hundreds of or thousands of base stations to cover a city while for
LPWAN only several base stations are needed to achieve similar performance. For LPWAN, though they all have large coverage, the range is a little different due to the protocol in detail. Besides, the flexible network establishment is another considered element. As discussed previously, LoRaWAN could be established conveniently by deploying LoRaWAN gateways. Sigfox and NB-IoT end devices need to be deployed based on service (base station location) from Internet Service Provider (ISP). The flexible network establishment could bring stable communication of good quality for some applications while for others this function may not be useful.

\section{E. Scalability}

Supporting thousands of end devices is a common feature of Sigfox, LoRaWAN, and NB-IoT. As the design objective, these LPWAN technologies provide high scalability. This results from the topology structure and operation mechanism of LPWAN. For instance, strategies like optimal channel selection make the same available channels to accommodate more communications. For applications with limited users, scalability is not so important because there always are available channels. However, for the large-scale scenarios, the scalability limitation could influence the performance of LPWAN

\section{F. Security}

With security methods, the LPWAN could prevent the user's privacy from hacked. LoRaWAN supports the security by a unique 128 bit AES key (called AppKey) and a globally unique identifier (EUI-64-based DevEUI), both of which are used during the device authentication process [10]. For Sigfox, It uses the CTR encryption key in the AES128 algorithm mode, which is unique for each device. The air security of the uplink implements several mechanisms: a message counter for replay attack protection, AES-128 in $\mathrm{CBC}$ mode for authentication and integrity checking, and CRC-16 for error detection. NB-IoT inherits the security mechanisms for confidentiality and authentication from LTE networks. LTE provides symmetric encryption and signature mechanisms to prevent data leakage and uses SIM cards to authenticate and identify devices in the network.

\section{EXPERIMENTS AND RESULTS OF LPWAN MODELING}

In this part, the experimental setup and the evaluation results of LPWAN are introduced. The setup of parking sensors is shown in Fig. 1.

This test used parking detection sensors as a use case. The parking detection sensor could detect the occupied/free status of park lots and transmit the data through the LPWAN network. In this test, different parking detection sensors using three LPWAN techniques were applied. Based on the experiments, the best applicable technique was selected for this application. 


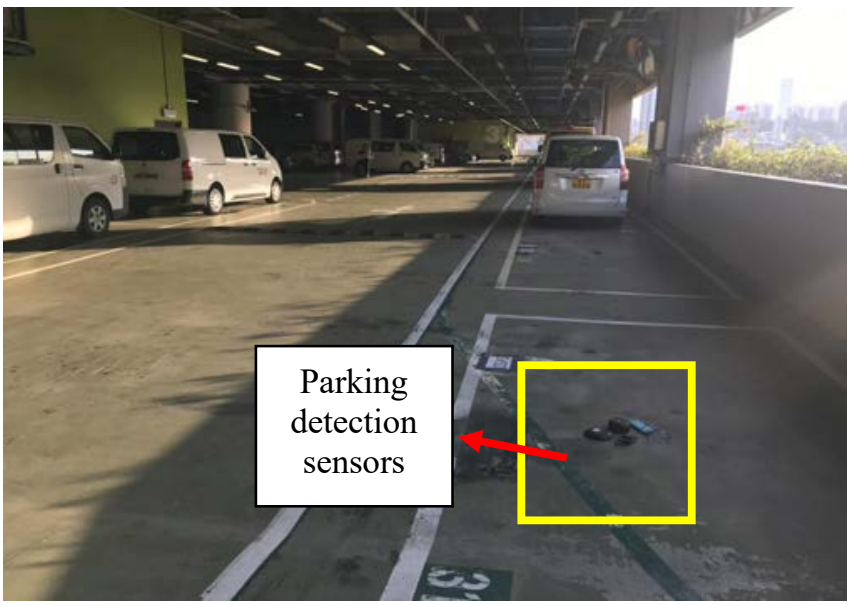

Fig. 1. The setup of parking detection sensors.

The evaluation matrices of the three IoT technologies are given in Table I. For this application, the weightings for six aspects are set as $30 \%, 2 \%, 20 \%, 30 \%, 5 \%, 13 \%$, respectively. Among them, latency, and coverage are distributed with more weighting considering their significance. The monitoring latency should be set as low as possible. The delayed messages may transmit incorrect information and confuse at peak hours. Sufficient coverage ensures the continuous operation of sensors. If the communication is in poor quality due to a weak signal, it is easy to lose important detection results. The aspect, power and cost, is given a weight as $20 \%$. Due to the large number, the power and cost of each parking detection sensor should be as low as possible so that it will not cause unaffordable spending. The following is security. It should be mentioned that the results in Table I only represent the performance of three LPWANs in this application rather than a general result. For other applications, new preferences and weightings should be set to satisfy requirements and achieve the best performance.

With these requirements in mind, the following technologies have been analyzed and compared following the captioned evaluation criteria.

TABLE I. THE EVALUATION MATRIC FOR AN ASSUMPTIVE APPLICATION BASED ON LP-INDEX

\begin{tabular}{|l|l|l|l|l|}
\hline Item & Weighting (\%) & LoRaWAN & Sigfox & NB-IoT \\
\hline Latency & 30 & 4 & 2 & 5 \\
\hline Data Capacity & 2 & 2 & 1 & 5 \\
\hline Power and Cost & 20 & 5 & 4 & 3 \\
\hline Coverage & 30 & 4 & 5 & 1 \\
\hline Scalability & 5 & 4 & 4 & 5 \\
\hline Security & 13 & 5 & 4 & 5 \\
\hline LP-INDEX & $100 \%$ & 4.29 & 3.64 & 3.4 \\
\hline
\end{tabular}

* The number (1-5) represents the ranking from low to high.
In summary, based on LP-INDEX, LoRaWAN is considered to be the best network to be applied in the parking detection sensor application.

\section{CONCLUSION AND FUTURE WORK}

To explore how to select the most suitable LPWAN techniques in different applications, we proposed LP-INDEX to address this challenge. The work leads as a research directive, which could provide guidelines for similar situations in establishing a smart city. The LP-INDEX considers the best practice of LPWAN in terms of six aspects according to the specific requirements of the applications comprehensively with reasonable weightings. A parking detection sensor testing was implemented as a use case to illustrate how LP-INDEX works. In the future, more details of LP-INDEX will be discussed and complemented.

\section{REFERENCES}

[1] Centenaro, Marco, et al. "Long-range communications in unlicensed bands: The rising stars in the IoT and smart city scenarios." IEEE Wireless Communications 23.5 (2016): 60-67.

[2] Raza, Usman, Parag Kulkarni, and Mahesh Sooriyabandara. "Low power wide area networks: An overview." IEEE Communications Surveys \& Tutorials 19.2 (2017): 855-873.

[3] Sigfox, "Sigfox connected objects: Radio specifications", [Online]. Available:

https://storage.sbg.cloud.ovh.net/v1/AUTH_669d7dfced0b44518cb1 86841 d7cbd75/prod_medias/b2be6c79-4841-4811-b9ee61060512ecf8.pdf

[4] U. Raza, P. Kulkarni, "Low Power Wide Area Networks: An Overview", IEEE Communications Surveys \& Tutorials, Vol. 19, No. 2, 2017, pp. 855-873

[5] Vodacom and Vodafone, "White paper: Narrowband-IoT: pushing the boundaries of IoT systems". [Online]. Available: https://www.vodacombusiness.co.za/cs/groups/public/documents/do cument/

[6] Crys, T. A. N., and T. A. N. Hwee-Pink. "Evaluation of Sigfox LPWAN for sensor-enabled homes to identify at risk community dwelling seniors." 2019 IEEE 44th Conference on Local Computer Networks (LCN). IEEE, 2019.

[7] Mekki, Kais, et al. "Overview of cellular LPWAN technologies for IoT deployment: Sigfox, LoRaWAN, and NB-IoT." 2018 ieee international conference on pervasive computing and communications workshops (percom workshops). IEEE, 2018.

[8] Mermer, Gültekin Berahan, and Engin Zeydan. "A comparison of LP-WAN technologies: An overview from a mobile operators' perspective." 2017 25th Signal Processing and Communications Applications Conference (SIU). IEEE, 2017.

[9] Wu, Chung Kit, et al. "Supply chain of things: A connected solution to enhance supply chain productivity." IEEE Communications Magazine 57.8 (2019): 78-83.

[10] LoRaWAN Security Whitepaper, "LoRaWANTM SECURITY". [Online]. Available: https://lora-alliance.org/sites/default/files/201905/lorawan_security_whitepaper.pdf 